\title{
La Mortalidad por Tuberculosis en El Puerto de Santa María (1923-1926)
}

\section{Tuberculosis mortality in El Puerto de Santa María (1923-1926)}

\section{A mortalidade por tuberculose em Puerto de Santa María (1923-1926)}

\author{
Irene López León ${ }^{1}$ J. César Muriel ${ }^{1}$ Ana M. Sáinz Otero ${ }^{2}$ \\ ${ }^{1}$ Diplomado Universitario en Enfermería por la Universidad de Cádiz. \\ ${ }^{2}$ Doctora por la Facultad de Medicina de la Universidad de Cádiz. \\ Cómo citar este artículo en edición digital: López León ,I., Muriel, J.C., Sáinz Otero, A.M. (2014) La Mortalidad por Tuberculo- \\ sis en El Puerto de Santa María (1923-1926). Cultura de los Cuidados (Edición digital) 18, 38. \\ Disponible en: http://dx.doi.org/10.7184/cuid.2014.38.10> \\ Correspondencia: Irene López León.c/ Almirante Faustino Ruiz n4, 5B. 11100 San Fernando \\ Recibido: 09/10/2013; Aceptado: 10/12/2013 \\ Correo electrónico: irene.lopezleon@alum.uca.es
}

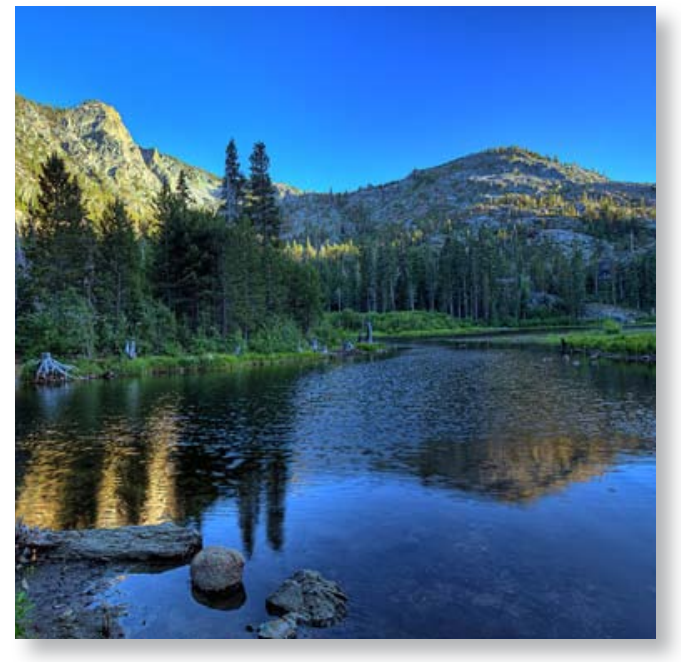

ABSTRACT

The main cause of mortality in El Puerto de Santa María (Cádiz) between 1923 and 1926 was tuberculosis. Our aim is to analyse the tuberculosis mortality rate in this town between 1923 and 1926 and to link it to the principal epidemiological determinants in order to describe the health and sanitary conditions in those years.

Descriptive and longitudinal study of the tuberculosis mortality in El Puerto de Santa María between 1923 and 1926. Our information sources were the entries in the register of deaths. A statistical analysis of the variables was carried out (death cause, sex, age...), with the programme SPSS15.0. The total amount of deaths caused by tuberculosis was 217 (13,7 $\%$ ) and the specific mortality rate was 28,22 in 10,000 inhabitants. 54\% (117) were males and 46\% (100) females. We can conclude:

1. Tuberculosis was the first cause of death in El Puerto between 1923 and 1926.

2. The tuberculosis mortality standards are similar in the rest of the province of Cadiz.

3. It would be necessary to perform a deeper study to know whether the cause of the decrease in the mortality rate in 1926 was a consequence of an improvement in health and sanitary policies.

Key words: Mortality, Tuberculosis, Historical Demography, Epidemiology.

\section{RESUMO}

A principal causa de mortalidade em Puerto de Santa María (Cádiz) entre os anos de 1923 e 1926 é a tuberculose. O objetivo é analisar a mortalidade por tuberculose nesta localidade durante o período de 1923 a 1926, relacionando-a com os principais determinantes epidemiológicos, contribuindo para a 
interpretação da realidade sanitária da época.

Trata-se de um estudo descritivo, longitudinal, da mortalidade por tuberculose em Puerto de Santa María de 1923 a 1926. As fontes foram os registos de óbitos do Registo civil. Realizou-se uma análise estatística das variáveis (causa de morte, sexo, idade...), com o programa SPSS 15.0. O total de óbitos por tuberculose foi de $217(13,7 \%)$ e a Taxa de Mortalidade específica de 28,22 por dez mil habitantes. Relativamente ao sexo, 54\% (117) correspondem a homens e $46 \%$ (100) a mulheres. As conclusões são as seguintes:

1. A tuberculose é a primeira causa de mortalidade em Puerto de Santa María entre 1923-1926.

2. O padrão de tuberculose na localidade é superior ao do resto da província.

3. É necessária uma série temporal mais ampla para poder atribuir a causa da descida dos óbitos em 1926 à melhoria das condições higiénico-sanitárias derivadas da atuação institucional.

Palavras-Chave: Mortalidade, Tuberculose, Demografía Histórica, Epidemiología.

\section{RESUMEN}

La principal causa de mortalidad en El Puerto de Santa María (Cádiz) durante 19231926 es la tuberculosis. El objetivo es analizar la mortalidad por Tuberculosis en dicha localidad durante 1923 a 1926, relacionándola con los principales determinantes epidemiológicos, contribuyendo a la interpretación de la realidad sanitaria de la época.

Estudio descriptivo, longitudinal de la mortalidad por tuberculosis en El Puerto durante 1923 a 1926. Las fuentes fueron los libros de Actas de defunción del registro civil. Se realizó el análisis estadístico de las variables (causa de defunción, sexo, edad...), con el programa SPSS 15.0. El total de defunciones por tuberculosis fueron $217(13,7 \%)$ y la Tasa de Mortalidad específica de 28,22 por diez mil habitantes. Atendiendo al sexo, el 54\% (117) corresponden a hombres y el 46\% (100) a mujeres. Las conclusiones son:

1. La Tuberculosis es la primera causa de mortalidad en El Puerto entre 1923-1926.

2. El patrón que sigue la mortalidad por tuberculosis en El Puerto es superponible al del resto de la provincia.

3. Es necesario una serie temporal más amplia para poder asignar la causa del descenso de las defunciones en 1926 a las mejoras de las condiciones higiénico-sanitarias derivadas de la actuación institucional.

Palabras Clave: Mortalidad, Tuberculosis, Demografía Histórica, Epidemiología.

\section{INTRODUCCIÓN}

En la provincia de Cádiz, a principios del siglo $\mathrm{XX}$, se recogen las cifras más altas de mortalidad por tuberculosis, en comparación con el resto de provincias españolas (Sáinz, 2008) (Sáinz, 2006) (Herrera, 2007). A pesar de las excelentes condiciones climáticas (luz, sol y mar), coexisten otros factores negativos, como son: la gran humedad existente, las malas condiciones sociales y económicas (hacinamiento, falta de ventilación y soleamiento de las calles y viviendas). Todo ello es señalado como "factores preparadores de la tuberculosis", haciendo hincapié algunos salubristas de la época en la importancia de las condiciones climatológicas. Es por ello que en 1908 se inician una serie de medidas encaminadas a disminuir dicha mortalidad y mejorar la calidad de vida de la población. Con anterioridad a esta fecha realizaron una labor infatigable en este sentido, entre otros, los médicos gaditanos 
Benito Alcina Rancé (1853-1902) y Bartolomé Gómez Plana (1860-1920) (Herrera, 2000). Medidas, fomentadas entre otros por el Dr. Leonardo Rodrigo Lavín (1867-1950) (Herrera, 2007), que promovió la lucha antituberculosa con sus escritos en la prensa gaditana, además de apoyar la creación del Centro Escolar de Investigaciones Biológicas, Dispensarios e Instituciones Antituberculosas y desarrollar tareas como: vacunación, reconocimiento de nodrizas, desinfecciones y análisis de alimentos y agua, renovación del alcantarillado e incluso la limpieza de calles con "agua salobre" y crecimiento de la ciudad en el extramuros.

En estos años de nuestro estudio, El Puerto de Santa María, como el resto del país, se encontraba en plena dictadura de D. Miguel Primo de Rivera; la ciudad tuvo que enfrentarse a problemas sociales y económicos como, por ejemplo, la deficiente cosecha de uva causada por las lluvias (Gatica, 2004), el encarecimiento de los precios de artículos de primera necesidad, como el pan, el carbón y las carnes (Gatica, 2004); y la crisis del sector naval gaditano, que produjo multitud de despidos (4000 obreros de la Factoría de Matagorda se quedaron sin empleo) (Gatica, 2004).

Podemos decir que las dos grandes lacras que afectaron a El Puerto de Santa María en este periodo fueron la mortalidad infantil y la muerte por tuberculosis (Herrera, 2000). La implantación del Reglamento de Sanidad Provincial (Marset, 1997) en 1925, aportaría las consiguientes mejoras en materia de higiene, vivienda, política sanitaria de profilaxis y condicionamiento de los centros sanitarios. Por aquel entonces la población portuense podía recibir atención sanitaria principalmente en el Hospital de San Juan de Dios (González, 1997), institución dependiente de la beneficencia, que era el principal hospital de la ciu- dad para las personas sin recursos. No descartamos que algunos pacientes fueran tratados en el Hospital de Mora (Herrera, 2008), de la beneficencia provincial, y que en verano algún niño portuense enfermo fuera enviado para su recuperación al sanatorio marítimo de Santa Clara (Herrera, 1989) en Chipiona.

El objetivo general que nos planteamos en esta comunicación es analizar la mortalidad por Tuberculosis en el Puerto de Santa María durante los años 1923 al 1926, relacionándola con los principales determinantes epidemiológicos y contribuir así a la interpretación de la realidad sanitaria de la época.

\section{MATERIAL Y MÉTODO}

Estudio descriptivo y longitudinal de la mortalidad por Tuberculosis en El Puerto de Santa María (Cádiz) durante los años 1923 al 1926, basado en el análisis estadístico de los registros de defunción. Las fuentes utilizadas fueron los libros de Actas de defunción del registro civil de dicha localidad, tomos 52, 53 y 54 .

La población censada en El Puerto de Santa María para los años 1923, 1924,1925, y 1926 son respectivamente 19.097, 19180,19.266 y 19.353. La Población abarcada para esta comunicación son todas las defunciones inscritas en el Registro Civil de El Puerto de Santa María, desde el 1 de enero de 1923 hasta el 31 de diciembre de 1926; ascendiendo a un total de 1584 defunciones y siendo 217 por Tuberculosis. Se incluyeron todos los casos en los cuales la causa de defunción fuera la tuberculosis; al tratarse de registros de defunción de los años 1923-1926 la nomenclatura de la época para el diagnostico de causas de defunción difiere del actual, por lo que se apuntan acepciones diagnosticas de tuberculosis tales como: "granulia", "hemoptisis", "mal de pott", "tisis" y "tabes dorsal". 
El análisis estadístico se realizó con el programa SPSS 15.0, donde se elaboró una base de datos en la que se recogen las variables a medir (sexo, mes y año de defunción, edad, causa de defunción) y un registro que se corresponde con cada uno de los fallecidos. Se obtuvo la distribución de frecuencias de las variables por mensualidades, anualidades y para el período completo.

\section{RESULTADOS}

Tras nuestra recogida de datos en el Registro Civil de El Puerto de Santa María, obtuvimos unas cifras en valores absolutos de 1584 defunciones para el periodo comprendido entre 1923 y 1926.

$\mathrm{Al}$ analizar la mortalidad, observamos que la principal causa de muerte en este periodo es la Tuberculosis, con una cifra de 217 defunciones; es decir, un porcentaje de $13,7 \%$ y una Tasa de Mortalidad específica de 28,22 por diez mil habitantes, siendo esta inferior siempre a la de la Capital gaditana. (Grafico1)

Supuestamente al tratarse de una enfermedad infecciosa deberíamos observar un comportamiento estacional, circunstancia que no se cumple (gráfico 2); se mantiene constante durante todo el periodo, con dos picos elevados en noviembre de 1924 y en enero de 1926 y dos descensos, uno en marzo de 1923 y otro en septiembre de 1925.

En cuanto a la mortalidad ligada al sexo, el 54\% de las defunciones (117) ocurridas en el periodo completo corresponden a hombres y el 46\% (100) a las mujeres. La edad media de defunción por tuberculosis en El Puerto de Santa María para el total del periodo es de 34 años.

Si observamos el gráfico 3 , los rangos de edad con mayor número de defunciones se dan en los adultos jóvenes. En primer lugar encontramos a los adultos de entre 21-30 años (23\%), seguido de los de 31-40 años (18\%) encontrándose en tercer lugar los jóvenes de 11-20 años (16\%). En los extremos del gráfico podemos encontrar como los menores de un año (2\%) y los mayores de 61-70 años (6\%) y 71-80 años (2\%) son los rangos de edad con menor número de defunciones. Si diferenciamos en cuanto al género, observamos un mayor número de defunciones en las mujeres con edades comprendidas entre 11 y 30 años, invirtiéndose los valores para las edades entre 31 y 70 años.

\section{DISCUSIÓN}

La principal causa de mortalidad para El Puerto de Santa María en este periodo la constituye la tuberculosis, que presenta una tasa de 28,22 por diez mil habitantes, cuando la tasa en la capital gaditana (Sáinz, 2008) aumenta hasta 40,51 por diez mil y la tasa a nivel nacional (Pascua, 1935) es del 15,5 por diez mil habitantes, encontrándose la ciudad portuense entre ambas (Molero, 1989).

La mortalidad por tuberculosis es prácticamente igual para los años del periodo, exceptuando 1924 donde aumenta hasta 60 defunciones. L. Rodrigo Lavín indica que las condiciones ambientales, sociales y económicas de las ciudades costeras son los factores que propician el desarrollo de esta enfermedad (Herrera, 2000). Dichas condiciones hacen que las enfermedades infecciosas, como la tuberculosis, tengan un claro comportamiento estacional, pero durante los años 1923 y 1926 no observamos estacionalidad ya que se mantiene constante durante todo el periodo. Cabe destacar una ligera disminución y estabilización en el número de defunciones en 1926 coincidiendo con la entrada en vigor del reglamento de Sanidad Provincial de 1925. 
Si analizamos las defunciones y el género, observamos, en cifras generales, que fallecen más hombres que mujeres; siendo el número de defunciones más elevado en mujeres en edad reproductiva (de 11 a 30 años). Según la OMS (OMS, 2011), la tuberculosis es una enfermedad que afecta mayoritariamente a mujeres en edad reproductiva, pero causa más defunciones en hombres, en edad adulta. Curiosamente si analizamos los datos de L. Rodrigo Lavín, observamos que en el Dispensario Victoria Eugenia de Cádiz se atendieron a 47 mujeres y 18 hombres (Herrera, 2004). Este dato nos resulta llamativo, ya que podría depender de factores como son la alimentación, el embarazo, parto y puerperio. Es por ello que lo consideramos como una posible línea de investigación abierta para el futuro.

\section{CONCLUSIONES}

La Tuberculosis es la primera causa de mortalidad en El Puerto de Santa María entre 1923 y 1926.

El patrón que sigue la mortalidad por tuberculosis en El Puerto de Santa María es superponible al del resto de la provincia.

Es necesario una serie temporal más amplia para poder asignar la causa del descenso de las defunciones en 1926 a las mejoras de las condiciones higiénico-sanitarias derivadas de la actuación institucional.

\section{BIBLIOGRAFÍA}

- Gatica Gote, D. (2004) Dictadura (y dictablanda) en El Puerto de Santa María, 1923-1931, Concejalía de Cultura del Ayuntamiento de El Puerto de Santa María, 26-28.

- González Márquez, D. (1997) Estudio Histórico Sanitario del Hospital de San Juan de Dios en El Puerto de Santa María (1793-1935). (Trabajo dirigido Herrera F.). Revista de Historia de El Puerto 19, 39-59.

- Herrera, F., Doña, F. (1989) Manuel Tolosa Latour (1857-
1919): impulsor de los sanatorios marítimos para la infancia. Anales de la Real Academia de Medicina y Cirugía de Cádiz XXV, 1, 89-97.

- Herrera, F. (2000) La situación sanitaria en la provincia de Cádiz en el siglo XX. En: Ramos Santana A. (Coord.). Cádiz. La Provincia en el siglo XX. Cádiz: Fundación provincial de Cultura.

- Herrera, F. (2000) Gavilla de Médicos Gaditanos. Quorum Editores.

- Herrera, F. (2007) La obra Sanitaria de Leonardo Rodrigo Lavín (1867-1850), Servicio de publicaciones de la Universidad de Cádiz, 67-82.

- Herrera, F. (2008) Archivos y hemerotecas: Espacios para la investigación de la Historia de la Enfermería. Híades. Revista de Historia de la Enfermería 10, 86-93.

- Marset, P., Rodríguez, E., Sáez, M. (1997) La Salud Pública en España. En: Martínez F y cols. Salud Pública. Madrid: McGraw-Hill-Interamericana, 1-24.

- Molero, J. (1989) La Tuberculosis como enfermedad social en los estudios epidemiológicos españoles anteriores a la Guerra Civil. Rev. Dynamics, vol. 9: 185-223.

- Organización Mundial de la Salud (OMS). Tuberculosis y género. Disponible en: http://www.who.int/tb/challenges/gender/page_1/es/index.html [consultado en septiembre de 2011].

- Pascua, M. (1935) Mortalidad específica en España. Madrid: Publicaciones Oficiales de la C.P.I.S., 11-14.

- Sáinz, AM., Herrera, F., Almenara, J., Lagares, C. (2006) La Mortalidad por enfermedades infecciosas en Cádiz (1924-1925). En: Pérez-Bustamante JA et al. (dirs: actas del IX Congreso de la Sociedad española de Historia de la Ciencias y de las Técnicas (Cádiz, 27-30 de septiembre de 2005). SEHCIT. Cádiz, 2006; tomo 1, 427-440.

- Sáinz, AM. (2008) La Mortalidad en Cádiz (1923-1929), [Tesis doctoral]. Cádiz: Servicios de Publicaciones de la UCA. 


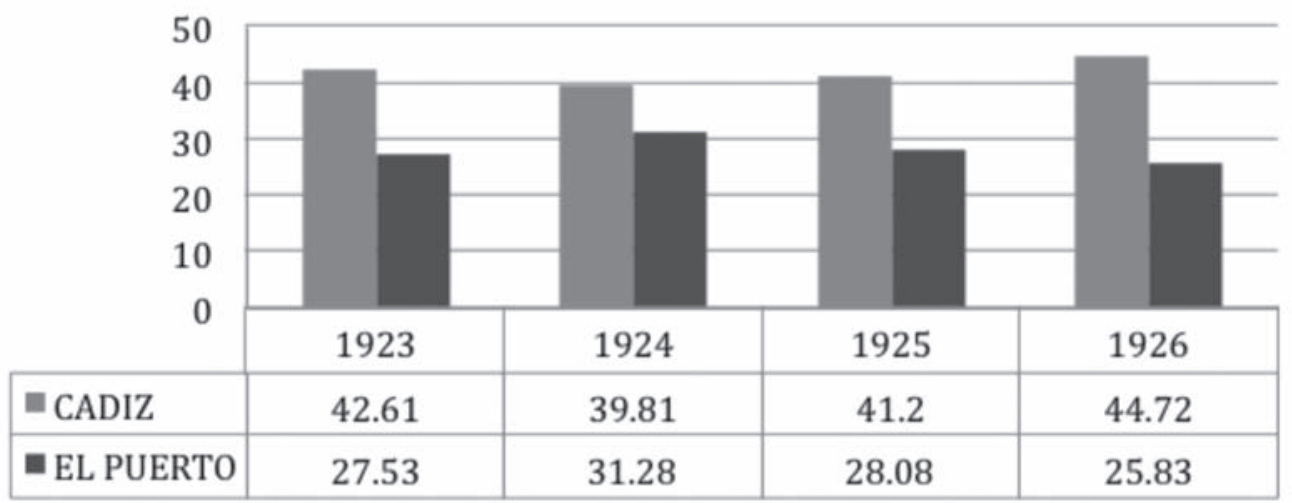

Gráfico 1. Tasa de Mortalidad Específica por Tuberculosis. Cádiz y El Puerto de Santa María 1923-1926. Fuente: Registro civil de Cádiz y El Puerto de Santa María. Elaboración propia 2011

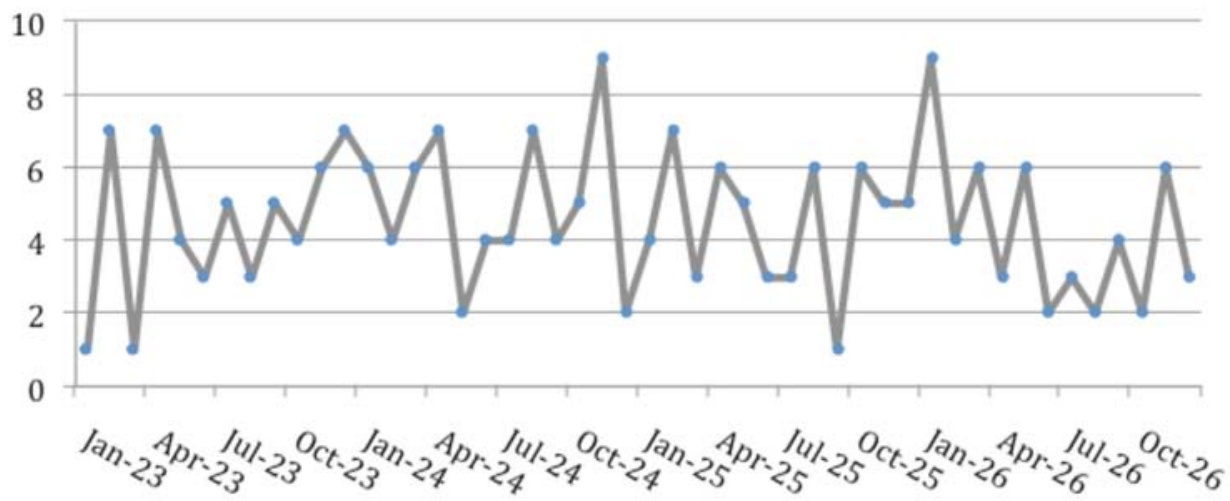

Gráfico 2. Evolución de la mortalidad por Tuberculosis. El Puerto de Santa María 1923-1926. Fuente: Registro civil de El Puerto de Santa María. Elaboración propia 2011.

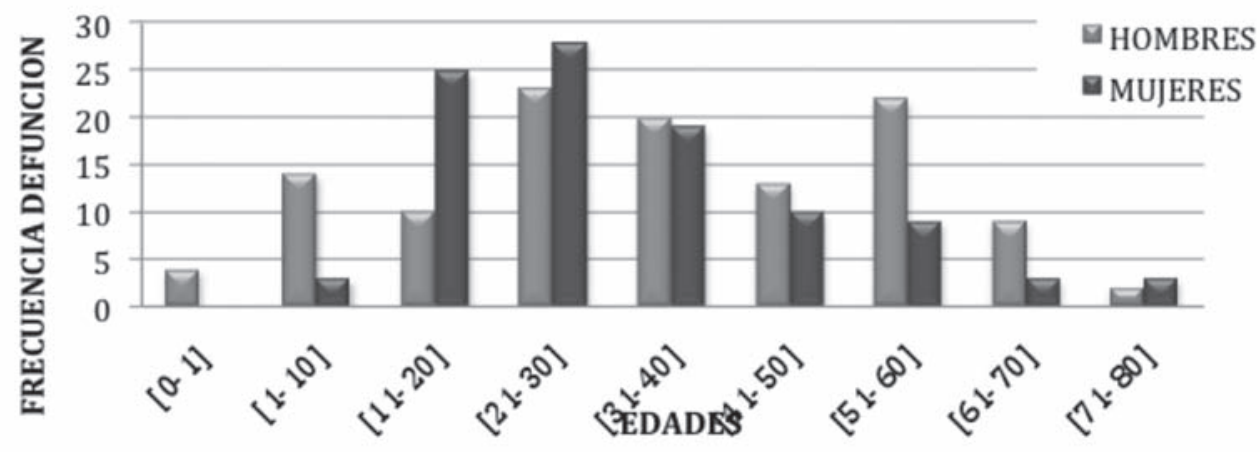

Gráfico 3. Distribución de Defunciones por Edad y Sexo. El Puerto de Santa María 1923-1926. Fuente: Registro civil de El Puerto de Santa María. Elaboración propia 2011. 